\title{
La cultura material en las literaturas y culturas iberoamericanas de hoy
}

\section{Héctor Hoyos}

STANFORD UNIVERSITY, ESTADOS UNIDOS

Profesor asociado del Departamento de Culturas Iberoamericanas de

la Universidad de Stanford y PhD de la Universidad de Cornell, ambas en Estados Unidos. Es autor del libro Beyond Bolaño: The

Global Latin American Novel (Columbia University Press, 2015), y coeditor del dosier "Theories of the Contemporary in South

America" para la Revista de Estudios Hispánicos (2014). Otros

artículos suyos aparecen en Third Text, Novel, Chasqui y Revista

de Estudios Sociales. Correo electrónico: hoyos@stanford.edu

\section{Cómo citar este texto:}

Hoyos, Héctor. "La cultura material en las literaturas y culturas

iberoamericanas de hoy". Cuadernos de Literatura 20.40 (2016): 254-

261. http://dx.doi.org/10.11144/Javeriana.cl20-40.cmlc

doi:10.11144/Javeriana.cl20-40.cmlc 
EL PRESENTE D OSS IER es fruto de la colaboración sostenida de un grupo de estudiosos de la literatura contemporánea, de múltiples nacionalidades y diversas trayectorias académicas, afincados en instituciones de Estados Unidos y Europa occidental. La composición de este grupo, algo azarosa y bastante fructífera, se conviene con su objeto de estudio, que es la globalización y sus efectos en la literatura y cultura actuales, principalmente en lengua castellana. El grupo se ha propuesto construir un aparato crítico y bibliográfico de literatura contemporánea en tiempo real. Para ello, desarrolla varias líneas de investigación, pues los autores, de diversas generaciones, se desempeñan dentro del campo literario de múltiples maneras que incluyen, entre otras, la reflexión teórica e historiográfica, la gestión cultural y los estudios de traducción.

Un primer encuentro, que tuvo lugar en la École Normale Supérieure, en 2013, coordinado por Gustavo Guerrero y Gersende Camenen, se concentró en los aspectos geográficos y espaciales de esta problemática. Al año siguiente, durante unas jornadas en la Universidad de Berna, lideradas por Catalina Quesada, el problema central fue el de la temporalidad; las ponencias de aquel encuentro las editó Erica Durante en un volumen con prólogo de Rodrigo Rey Rosa (2015). Es un gusto dar a conocer en Cuadernos de Literatura versiones corregidas y aumentadas de las ponencias que se presentaron en un tercer coloquio, convocado por Aníbal González, que se sostuvo en la Universidad de Yale el 5 y 6 de marzo de 2015. ${ }^{1}$

El punto de partida para nuestra reflexión fue constatar las estrechas relaciones entre globalización y cultura material. La intensificación en el comercio multilateral de bienes e ideas, que se agudiza desde 1989 por efecto de la creciente integración de las economías del mundo y de la adopción de nuevas tecnologías de comunicación, trae consigo un nuevo régimen en nuestra relación con los objetos. Los viajeros descubren que resulta más difícil llevar un obsequio del país que no esté ya disponible en el lugar de destino. Los estudiosos de la cultura que nos formamos leyendo las fotocopias de otros, $y$ las fotocopias de sus fotocopias, descubrimos que los libros existen, y aunque sigan siendo inmoderadamente costosos, pueden adquirirse a través de internet, modificando de paso las dinámicas de la edición local. Invitar a escritores y académicos extranjeros a intercambiar ideas, de ser un acontecimiento excepcional, poco a poco empieza a hacerse

1 Agradecemos el apoyo de todas las instituciones y personas que hicieron posible tanto el encuentro como la edición de este dosier, que son más de la que es posible mencionar aquí. A título personal, agradezco especialmente a la Fundación Alexander von Humboldt y a mis anfitriones durante una estancia de investigación en la Universidad Libre de Berlín, los profesores Joachim Küpper y Susanne Zepp. Agradezco también a Caroline Egan por asistir en la preparación del dosier. 
normal; lo mismo sucede en el sentido opuesto. Estos ejemplos, entre muchos otros posibles, sugieren que hay una dimensión "palpable" de la experiencia de la globalización que debe pensarse desde los estudios literarios y culturales.

La cultura material es un concepto fundamental de la antropología moderna (Buchli). Al invocarlo aquí hacemos un uso libre del término. No nos interesa, mediante el estudio de los objetos de una cultura ajena, tratar de reconstruir la cosmología o las costumbres de un pueblo. Antes bien, una consideración perspicua de las cosas y prácticas materiales de la propia cultura, tamizados por la creación literaria, constituye nuestro objeto de estudio. A partir del trabajo de Bruno Latour y Jane Bennett, entre otros pensadores que contribuyen a la heterogénea corriente que se ha dado en llamar nuevo materialismo, consideramos los objetos cocausantes de fenómenos en los que también participamos los humanos (Coole y Frost). En otras palabras, queremos desplazarnos de una ontología que separa sujeto y objeto, cuyo ejemplo paradigmático es Descartes, a una que reconoce las propiedades agénticas de cosas que, aunque carecen de intención, sí tienen una efectividad en el mundo. Ese planteamiento afecta la literatura doblemente, por cuanto esta hace parte de procesos materiales y también los representa. A contrapelo de la teoría dualista de la mímesis, en la que la literatura y otras artes reflejarían lo material en lo espiritual, se abre paso un pensamiento más matizado sobre la compleja interacción interna entre creación y realidad.

Algunos precedentes de esta aproximación en la crítica literaria norteamericana son los trabajos de Bill Brown y Maurizia Boscagli; las condiciones materiales de la literatura hispanoamericana exigen un tratamiento distinto, que buscamos esbozar aquí. Se trata de un primer paso dentro de lo que anticipamos será una corriente influyente en nuestro campo. Entre otras cuestiones, el giro material contemporáneo permite repensar la mercantilización (commodification) de objetos y personas, las redes de intercambio de productos culturales, el libro como objeto de consumo y comercio, los soportes tangibles de la literatura, la materialización y desmaterialización de la cultura y, en un sentido amplio, los flujos de la globalización. En el doble registro antes embozado, permite además considerar a un tiempo la representación de procesos materiales y la materialidad de la escritura misma. En un sentido, esta corriente se alinea con planteamientos poshumanistas, por cuanto nuestra especie cede protagonismo a aquello que la rodea. Sin embargo, de manera dialéctica, podría decirse que en ese postantropocentrismo también está presente el ideal humanista de nihil a me alienum puto (nada me es extraño), extendido ahora a animales y cosas. Por otra parte, el trabajo, como categoría central del materialismo histórico, también merece un lugar dentro de esta discusión, que no es ajena por tanto al poder y a la política. 
Apenas comienza la tarea de articular las tradiciones materialistas y de examinar el rol de la literatura latinoamericana en esa encrucijada.

En el primer artículo de esta compilación, titulado "De la abundancia a la escasez: distopías latinoamericanas del siglo XXI", Eduardo Becerra interviene en la percepción del mainstream de lo contemporáneo en la literatura latinoamericana. A contrapelo del imaginario de abundancia que caracterizó la escritura más recordada de autores como Jorge Volpi o Alberto Fuguet, Becerra examina uno de escasez, presente en autores argentinos y mexicanos, que critica el sistema económico. En Pedro Mairal, Rafael Pinedo, Carlos González Muñiz y David Miklos, son los objetos descartados, en medio de paisajes distópicos y postapocalípticos, los que vienen a reavivar la función social de la narrativa. Mairal sigue siendo objeto de estudio en el artículo de Jesús Montoya, titulado "Hacia una arqueología del presente: cultura material, tecnología y obsolescencia". El crítico desarrolla la discusión sobre el simulacro de sus trabajos anteriores para comparar al autor con una representativa figura trasatlántica, Agustín Fernández Mallo. Según Montoya, el peculiar simulacro de estos escritores consigue un extrañamiento de los objetos cotidianos que vendría a configurar una perspectiva arqueológica sobre el presente.

En "Arqueologías globales en la literatura cubana: de las ruinas al chicle", Catalina Quesada da un giro a estas temáticas. Por obvias razones, la retórica de la abundancia que criticaba Becerra está ausente de la literatura de la isla en las últimas décadas; en cambio, una de la escasez ha venido, en clave realista, a asociarse a lo nacional. Quesada emplea de manera parecida a Montoya la figura de la arqueología, en este caso para discutir obras de Jorge Enrique Lage y Legna Rodríguez Iglesias. Para la crítica, estos autores dejarían de lado los objetos de la escasez con miras a construir una narrativa nacional paralela que se desmarca del realismo y de la carencia. La relación entre realismo y representación mimética de los objetos también se aborda en "Artistas y antropólogos: estéticas, objetos y diagnóstico cultural en la novela argentina contemporánea" por Teresa Orecchia Havas. La crítica se enfoca en el campo literario argentino, particularmente en la obra de Alan Pauls y Martín Kohan. Tras demostrar la afinidad de la escritura de estos autores con un proyecto estético como el del turco Orham Pamuk, quien explícitamente construye su narración en torno a objetos llenos de sentido, Orecchia Havas sitúa ese interés en la materialidad frente a las discusiones sobre realismo e influencia del arte contemporáneo que han ocupado la atención del campo en los últimos años.

La contribución de Erica Durante, titulada "Entre inseguridad y nomadismo: la maleta como símbolo y objeto de globalización" también parte de un 
referente no latinoamericano, la película Up in the Air (2009), de Jason Reitman, para construir una tipología de representaciones del equipaje en un conjunto amplio de narrativas iberoamericanas contemporáneas. Las observaciones de Durante no solo constituyen un topos, sino que describen el poder de mediación del objeto mismo, cuya "vida social", en términos de Appadurai, permite construir una narrativa de la globalización desde lo no humano. Mientras que Durante se concentra en un solo objeto y abre desde allí una perspectiva más amplia, aquella es ya el punto de Vicente Luis Mora en "Visión panorámica de la representación de objetos en la literatura hispánica reciente". El ensayo de Mora es de este modo un valioso aporte para los investigadores de las nuevas materialidades en las literatura en lengua castellana, tanto por el vasto corpus que traza como por las categorías de objeto aurático (en sentido benjaminiano) o proyectado (como foco de atención simbólica) que identifica.

Los artículos que siguen desarrollan en diversas direcciones las líneas de investigación de los anteriores. En "Cuerpos anfibios: metamorfosis y ectoentidad sexual en $X X Y$ de Lucía Puenzo", Moira Fradinger analiza la influyente película argentina contra el trasfondo de la reciente Ley de Identidad de Género de ese país. Mediante una rica especulación teórica en la línea de Donna Haraway, Fradinger muestra cómo las tortugas que aparecen en la película, cuyo sexo es determinado por el medioambiente, le permiten a Puenzo presentar la materia como indeterminada y no como indecidible (como la vería el giro lingüístico) o anterior a la cultura (como la vería un esencialismo biologicista). El modelo de ectoentidad sexual que propone la crítica se ajustaría mejor a sexualidades explayadas en toda la riqueza y variación de las formas vitales.

Por su parte, en su ensayo "Materialismo, realismo y prosaísmo en la poesía latinoamericana de los noventa", Gustavo Guerrero aborda la problemática de la relación entre las palabras y las cosas en un corpus de poetas jóvenes de distintos lugares de América Latina. A contrapelo de precedentes tan importantes como las poéticas de Ponge o de Eliseo Diego, afirma Guerrero, los poetas de la década privilegian "el silencio de las cosas". Como ilustra el crítico con amplios ejemplos textuales, ese desencanto viene a subvertir el valor de mercancía (commodity) conferido a los objetos del deseo en el neoliberalismo. La vitalidad de la poesía de los últimos años, género que lamentablemente no se ha estudiado con tanta atención como la prosa, se constata también en el artículo de Miguel Gomes, "Umbrales, remediaciones y cultura material en la poesía venezolana contemporánea". El crítico muestra cómo su objeto de estudio ha pretendido desbordar las posibilidades del lenguaje escrito en búsqueda de una expresión multimedial, que reclama una nueva materialidad para la poesía. Para Gomes, la 
interiorización contemporánea del performance, inspirada en el grupo El Techo de la Ballena de los años sesenta, sería una forma de intervenir contra un discurso oficial maniqueo y patriotero.

En la misma vena de analizar la materialidad del objeto libro, Florence Olivier presenta "De algunos usos de la fotografía en la literatura hispanoamericana contemporánea". Su artículo constata de qué manera, justo cuando la fotografía digital ha liberado al medio fotográfico de la impresión en papel, autores como La vida descalzo (2006) de Pauls, Memorial del engaño (2014) de Volpi y Perros héroes (2006) de Mario Bellatin recurren cada vez más a ella. Olivier reconstruye una fenomenología de la experiencia de lectura, atenta a los efectos de aletargamiento o proliferación de sentido que posibilita la fotografía. Por su parte, en "El oficio de traducir: de algunos traductores en la narrativa argentina de los años noventa y 2000", Gersende Camenen retoma la obra de Mairal, El año del desierto (2005), pero la sitúa en una constelación particular dentro de la literatura argentina de las últimas décadas, junto a El pasado de Pauls (2003), El Testamento de O'Faral de Marcelo Cohen (1995) y El traductor de Salvador Benesdra (1998). Las tres obras presentan un protagonista traductor y, como señala Camenen, una reflexión materialista sobre la escritura misma. La traducción, en cuanto mediación entre lenguaje y referente y entre materia y símbolo, sería así un elemento bisagra entre las políticas de la traducción (que describen Emily Apter y Martín Gaspar en sus respectivos registros) y el giro material contemporáneo.

Mi artículo "Materia prima e historia en Muñecas" se ocupa de la novela de Ariel Magnus (2008) para introducir la noción de materialismo transcultural: un modo narrativo que combina elementos del nuevo materialismo y de su variante histórica. En particular, constato de qué modo la novela articula trazos del extractivismo cauchero y del exterminio nazi, tanto enfocándose en los objetos como en su lugar dentro de procesos históricos. Así como para Hayden White el modernism permitía pensar otras maneras de contar la historia, el materialismo transcultural permitiría imaginar un neohistoricismo postantropocéntrico.

En el último artículo aquí reunido, "Entrando en materia: novela, poesía y cultura material en El ruido de las cosas al caer", Aníbal González lleva a cabo un tour de force en la recepción crítica del novelista bogotano Juan Gabriel Vásquez y una síntesis de varias de las líneas de investigación de este dosier. Tras un sucinto recorrido por los distintos regímenes de representación de objetos en la novela realista decimonónica y la del boom latinoamericano, González señala cómo Vásquez, más bien, sigue los cánones de la poesía, en particular la de José Asunción Silva y Aurelio Arturo. Tras señalar que el patrón predominante en la novela es el de "cosas que se mueven, gente que se inmoviliza", González muestra convincen- 
temente que los objetos de Vásquez se sumergen en una atmósfera de ensoñación que busca una "serena reconciliación" con la historia. Quedaría entonces abierta la puerta,justamente, para reflexionar sobre el rol de una aproximación materialista, en el horizonte histórico actual, frente a la conciliación o la denuncia. Sea ello como fuere, como oportunamente señala González, es el trabajo atento con la materialidad del lenguaje - voz, iteración, incluso rima - la que le permite a escritores como Vásquez "comunicar un mensaje urgente".

A propósito mencionaba antes el interés del presente dossier tanto por la literatura dentro de la cultura material como por la cultura material dentro de la literatura. Podría recorrerse este número de Cuadernos de Literatura pasando de un objeto global a otro: desde maletas perdidas en los aeropuertos del mundo a casetes con la grabación de un siniestro aéreo, desde chicles en Cuba hasta muñecas sexuales en Alemania. Y en el continuo entre lo orgánico y lo inorgánico, y entre cosas, animales y humanos, habría reptiles de sexo cambiante, traductores autores y libros de poemas que mutan en cómics. También son diversas las tradiciones académicas a las que pertenecen los críticos aquí reunidos, como es posible constatar por cuestiones de fondo o de forma. Ejemplo evidente de lo primero son los distintos acervos bibliográficos de que disponen los artículos; de lo segundo, el uso en unos ámbitos vigentes y en otros no del plural mayestático, entre otras convenciones de la escritura académica.

Mi labor como editor invitado ha sido preservar, antes que suprimir, esa diversidad. También en la crítica, como en la narrativa, se acompasan los espacios de la globalización; en lugar de imponer una economía del prestigio o de buscar centralizar criterios de validación académica, conviene una aproximación, por paradójico que suene, tanto rigurosa como flexible. (En su última época, la revista que acoge esta colección de artículos ha sabido construir un espacio dialógico de tales características.) Coincidimos, sin embargo, en señalar el valor de releer y estudiar a varios autores y obras recientes que así, poco a poco, van ganando densidad crítica. Podría decirse que en estas páginas se captura un Zeitgeist, si no fuera porque expresiones como el espíritu de los tiempos, tan valiosas como le han sido al humanismo, traen consigo muchas de las fracturas que el corpus estudiado busca, precisamente, reparar: entre alma y cuerpo, entre las palabras y las cosas.

\section{Obras citadas}

Appadurai, Arjun, ed. The Social Life of Things: Commodities in Cultural Perspective. Cambridge: Cambridge University Press, 1986. Impreso. 
Apter, Emily. Against World Literature: On the Politics of

Untranslatability. London/New York: Verso, 2013. Impreso.

Boscagli, Maurizia. Stuff Theory: Everyday Objects, Radical

Materialism. New York: Bloomsbury, 2014. Impreso.

Brown, Bill. A Sense of Things: The Object Matter of American Literature.

Chicago: University of Chicago Press, 2003. Impreso.

Brown, Bill, ed. Things. Chicago: University of Chicago Press, 2004. Impreso.

Buchli, Victor, ed. The Material Culture Reader. Oxford: Berg, 2002. Impreso.

Coole, Diana H. y Samantha Frost, eds. New Materialisms: Ontology, Agency, and Politics. Durham: Duke University Press, 2010. Impreso.

Durante, Erica, ed. Los meridianos de la globalización: estudios sobre el tiempo en la literatura latinoamericana contemporánea. Bruxelles: Presses Universitaires de Louvain, 2015. Impreso.

Gaspar, Martín. La condición traductora: sobre los nuevos protagonistas de la literatura latinoamericana. Rosario: Beatriz Viterbo, 2014. Impreso. 